\title{
Symmetry breaking in competing single-well linear-nonlinear potentials
}

\author{
Dmitry A. Zezyulin ${ }^{1}$, Mikhail E. Lebedev ${ }^{2,3}$, Georgy L. Alfimov ${ }^{2,4}$, and Boris A. Malomed ${ }^{5,1}$ \\ ${ }^{1}$ ITMO University, St. Petersburg 197101, Russia \\ ${ }^{2}$ Institute of Mathematics with Computer Center, Ufa Scientific Center, \\ Russian Academy of Sciences, Chernyshevskii str., 112, Ufa 450008, Russia \\ ${ }^{3}$ All-Russian Institute for Scientific and Technical Information, \\ Russian Academy of Sciences, 20 Usievich str, Moscow, 125190, Russia \\ ${ }^{4}$ Moscow Institute of Electronic Engineering, Zelenograd, Moscow, 124498, Russia and \\ ${ }^{5}$ Department of Physical Electronics, School of Electrical Engineering, Faculty of Engineering, \\ and Center for Light-Matter Interaction, Tel Aviv University, Tel Aviv 69978, Israel
}

(Dated: October 5, 2018)

\begin{abstract}
The combination of linear and nonlinear potentials, both shaped as a single well, enables competition between the confinement and expulsion induced by the former and latter potentials, respectively. We demonstrate that this setting leads to spontaneous symmetry breaking (SSB) of the ground state in the respective generalized nonlinear Schrödinger (Gross-Pitaevskii) equation, through a spontaneous off-center shift of the trapped mode. Two different SSB bifurcation scenarios are possible, depending on the shape of the nonlinearity-modulation profile, which determines the nonlinear potential. If the profile is bounded (remaining finite at $|x| \rightarrow \infty$ ), at a critical value of the integral norm the spatially symmetric state loses its stability, giving rise to a pair of mutually symmetric stable asymmetric ones via a direct pitchfork bifurcation. On the other hand, if the nonlinear potential is unbounded, two unstable asymmetric modes merge into the symmetric metastable one and destabilize it, via an inverted pitchfork bifurcation. Parallel to a systematic numerical investigation, basic results are obtained in an analytical form. The settings can be realized in Bose-Einstein condensates and nonlinear optical waveguides.
\end{abstract}

\section{INTRODUCTION}

External potentials, which steer the propagation and trapping of electromagnetic fields in photonics and matter waves in Bose-Einstein condensates (BECs), feature spatial symmetry in many physically important settings, a commonly known example being double-well potentials, which were explored in diverse physical settings [1, often in a combination with nonlinearity 22-20] (see also book 21]). It is well known too that the symmetry of the ground state in models including the self-focusing nonlinearity follows the symmetry of the underlying potential structure, which is always true in quantum mechanics 22] (and, more generally, in any linear theory), only as long as the nonlinearity remains weak enough. A generic effect, which sets in at a critical strength of the nonlinearity, is spontaneous symmetry breaking (SSB). In particular, in the framework of the nonlinear Schrödinger equation (NLSE), which is a generic model for many settings in optics and BEC, SSB was first considered in Ref. 23 (in terms of a discretized version of the NLSE, the SSB concept was introduced in another early work 24]). Generally, above the SSB point, the ground state of the one-dimensional (1D) NLSE model amounts to a soliton which is located at one of local minima of the underlying double- or multi-well potential structure. Because the particular minimum is chosen spontaneously among two or several available ones, the respective ground state features double or multiple degeneracy, which is possible in nonlinear systems, being forbidden in the linear Schrödinger equation [22].

In addition to the usual linear (in particular, doublewell) potentials, NLSE-based models may include non- linear pseudopotentials 25, which are represented by a spatially-dependent coefficient in front of the nonlinear term (we stress that the nonlinear interaction remains local, while its strength may become a function of coordinates). Pseudopotentials can be created in optics, by implanting nonlinearity-inducing dopants into the host medium 26, as well as in BEC, making use of a locally applied Feshbach resonance (FR), controlled by tightly focused laser beams. Several examples of spatial and spatiotemporal nonlinear pseudopotentials for BEC have been created in the experiment by means of the latter technique 27]-29]. Another possibility is the creation of an effective pseudopotential "painted" by a rapidly moving laser beam [30. Various soliton modes supported by pseudopotentials have been studied in detail (thus far, in the theoretical form) [31. In particular, double-well pseudopotentials based on the self-attractive nonlinearity give rise to specific SSB effects 32-34.

In this work we aim to demonstrate that the SSB is possible not only in double-well (pseudo)potentials, but also in a combination of competing linear and nonlinear single-well potentials, assuming that the former one is confining, while the nonlinear potential is expulsive. In terms of optics, this may be considered as a combination of a linear waveguide and nonlinear antiwaveguide. Previously, the opposite situation was considered, viz., competition of linear antiwaveguiding and nonlinear selffocusing, which produced not SSB effects, but transient regimes for quasi-stable propagation of spatial solitons [35. To the best of our knowledge, the SSB in the absence of a double-well structure was not demonstrated previously. Here, we find that, depending on the shape of the nonlinear potential, the SSB proceeds according to 
one of the two possible scenarios: in one case, the original symmetric ground state loses its stability through a direct bifurcation of a pitchfork type [36, which gives rise to a pair of stable asymmetric states; in the other case, the pitchfork bifurcation is inverted, leading to merger of two asymmetric states into a metastable symmetric one, leading to its destabilization.

The rest of the paper is organized as follows. In Sec. III. we describe the physical model. In Sec. III we present the main numerical results concerning the SSB in the competing linear-nonlinear potentials. Section IV offers the analytical treatment that supports the numerical results. Finally, Sec. $\mathrm{V}$ concludes the paper and provides an outlook on future research.

\section{THE MODEL}

We consider the 1D NLSE, written here as the GrossPitaevskii equation (GPE) which governs the evolution of the macroscopic BEC wave function $\Psi(x, t)$ [37]:

$$
i \Psi_{t}=-\Psi_{x x}+\frac{1}{2} \omega^{2} x^{2} \Psi-P(x) \Psi|\Psi|^{2},
$$

with time $t$ and coordinate $x$ scaled so that $\hbar=1$ and normalized atomic mass is $m=1 / 2$, whereas $\omega^{2}$ is the strength of the linear harmonic-oscillator (HO) trapping potential, and $P(x)>0$ determines the nonlinear pseudopotential, induced by the self-attractive nonlinearity, which we adopt in the form of a simple single-well structure:

$$
P(x)=1+A \tanh ^{2} x,
$$

with $A>0$. In BEC, this pseudopotential profile can be easily created by juxtaposing a local FR, induced by a focused laser beam, with the uniform self-attraction controlled by the uniform illumination, which can be readily implemented, e.g., in the condensate of ${ }^{174} \mathrm{Yb}$ atoms 28. As for the linear HO potential, it is a standard ingredient of any experimental setting dealing with BEC. In optics, the local-nonlinearity modulation profile defined by Eq. (2) can be made by uniformly doping the periphery of the waveguide with a resonant nonlinearity-enhancing material [26], while leaving the core area undoped.

As shown below, it is important that function $P(x)$ is bounded, i.e., its maximum value, $P(x= \pm \infty)=1+A$, is finite. Several other profiles $P(x)$ with shapes similar to one defined by Eq. (2) have also been considered, to check that the results reported below do not essentially depend on the specific choice of the bounded pseudopotential. This conclusion implies that the results are structurally stable, once they are not affected by a variation of the particular shape of the pseudopotential (obviously, in the actual experiment the shape can be designed with a finite accuracy).

The Hamiltonian corresponding to Eq. (1) is

$$
H=\int_{-\infty}^{+\infty}\left[\left|\Psi_{x}\right|^{2}+\frac{1}{2} \omega^{2} x^{2}|\Psi|^{2}-\frac{1}{2} P(x)|\Psi|^{4}\right] d x .
$$

It follows from here that the structure defined by Eq. (2) represents a repulsive nonlinear-potential barrier, as the strength of the local self-attraction has a minimum at $x=0$. Accordingly, the equilibrium position of a soliton in the present system is determined by the competition between the trapping linear $\mathrm{HO}$ potential and expulsive pseudopotential corresponding to Eq. (2). The subsequent analysis reveals the main finding of this work: the competition of the linear and nonlinear spatially symmetric potentials creates an asymmetric ground state, past the SSB point, in the absence of any double-well potential. We also demonstrate that switching from the bounded pseudopotential (2) to an unbounded one leads to a significantly different SSB scenario, with neither symmetric state nor asymmetric one being stable past the bifurcation point.

We look for stationary states with real chemical potential $\mu$ as $\Psi(x, t)=e^{-i \mu t} u(x)$, where $u(x)$ is a localized real stationary wave function which satisfies equation

$$
u_{x x}+\mu u-\frac{1}{2} \omega^{2} x^{2} u+P(x) u^{3}=0,
$$

and can be characterized by its norm (proportional to the number of atoms in the corresponding BEC), $N=\int_{-\infty}^{+\infty} u^{2}(x) d x$. Symmetric (even) and antisymmetric (odd) solutions are defined, respectively, by $u(x)=$ $u(-x)$ and $u(x)=-u(-x)$, whereas for asymmetric modes one has $|u(x)| \neq|u(-x)|$.

\section{NUMERICAL RESULTS}

Symmetric and symmetry-broken states can be found numerically, applying the standard iterative Newton's method, with a properly chosen initial guess, to Eq. (4). Since in this work we are interested in fundamental nodeless solutions, we used the initial guess in the form of Gaussian profiles $u_{0}(x)=a \exp \left(-\left(x-x_{0}\right)^{2}\right)$, where $a$ and $x_{0}$ are the trial parameters (obviously, $x_{0}=0$ should be chosen if the target solution is symmetric, and $x_{0} \neq 0$ otherwise). If the Newton's method converges to a stationary solution for some $\mu$, a family of nonlinear modes can be found by means of the continuation in $\mu$. To characterize the SSB bifurcation, we introduce the center-ofmass coordinate,

$$
X_{c}=N^{-1} \int_{-\infty}^{+\infty} x u^{2}(x) d x
$$

which is zero for symmetric and antisymmetric solutions, and nonzero for asymmetric ones.

The resulting bifurcation diagrams for the bounded pseudopotential (2) are displayed in Fig. 1. As follows from Fig. 1 (a,b), a direct bifurcation of the pitchfork type [36] occurs, with the increase of $N$, at the critical point, $N=N_{\text {cr }}$, as shown in panel (c): two asymmetric states, with $X_{c}>0$ and $X_{c}<0$, branch off from the symmetric one, with $X_{c}=0$, at $N>N_{\mathrm{cr}}$. Because the governing 
equation (1) keeps the global symmetry, i.e., it is invariant with respect to the space reflection, $x \rightarrow-x$, asymmetric solutions always emerge in two mutually-mirrored copies, i.e., $u(x)$ and $u(-x)$ with identical norms, therefore states $u(x)$ and $u(-x)$ are indistinguishable in the $(N, \mu)$-diagram plotted in Fig. 1(b). The SSB bifurcation generates asymmetric states above a critical value of the norm, $N>N_{\text {cr }}$. At $\mu \rightarrow 1$, the norm $N$ is vanishing, and the symmetric mode transforms into the harmonically trapped linear one with an infinitely small amplitude. Another visualization of the SSB bifurcation is presented in Fig. 11(c), in the $\left(X_{c}, N\right)$ plane.

Dependence of critical norm $N_{\text {cr }}$ at the SSB point on depth $A$ of the modulation of pseudopotential (2) is the most important characteristic of the setting under the consideration. The numerically found dependence is plotted in Fig. 1(d), where one observes that $N_{\text {cr }}$ slowly decays at $A \rightarrow \infty$, and $N_{\text {cr }}$ diverges as $A$ decreases, in agreement with the well-known fact that SSB does not occur in the model combining the linear HO potential and spatially uniform self-attractive nonlinearity [10, 13, 41. These asymptotic features can be easily explained analytically. Indeed, in the case of very large $A$, term 1 in definition (2) for $P(x)$ may be neglected, which allows one to remove $A$ by rescaling, leading to an asymptotic relation valid for $A \rightarrow \infty$ :

$$
\left(N_{\mathrm{cr}}\right)_{A \rightarrow \infty}=\text { const } \cdot A^{-1} \text {. }
$$

Profiles of symmetric and asymmetric modes coexisting at the same value of chemical potential $\mu$ are plotted in Fig. 1(e) and Fig. 1(f), respectively. Both solutions have the nodeless single-peak shape, but the maximum of the symmetric mode is located exactly at $x=0$, whereas the maximum of the asymmetric one is shifted to $x>0$, the mirrored asymmetric solution having its maximum shifted to $x<0$.

Next, we address the linear stability of the found symmetric and asymmetric solutions. First, Fig. 11(b) clearly shows that both the symmetric and asymmetric branches satisfy the necessary stability condition in the form of the Vakhitov-Kolokolov (VK) criterion, $d N / d \mu<0 \quad 38$ 40. Then, following the standard procedure, the stability problem amounts to the evaluation of eigenvalues of the linearization operator $\mathcal{L}=\mathcal{L}_{+} \mathcal{L}_{-}$, where (see, e.g., book 21] for details)

$$
\mathcal{L}_{ \pm}=\frac{d^{2}}{d x^{2}}+\mu-\frac{1}{2} \omega^{2} x^{2}+(2 \pm 1) P(x) u^{2}
$$

Mode $u(x)$ is linearly stable if all eigenvalues of $\mathcal{L}$ are real and positive. If a negative or complex eigenvalue $\Lambda$ is found in the spectrum of $\mathcal{L}$, then $u(x)$ is unstable, with instability growth rate $|\operatorname{Im} \sqrt{\Lambda}|$. Numerically computing the eigenvalues for the symmetric and asymmetric states, we observe that the symmetric branch is stable at $\mu>\mu_{\text {cr }}$ (in other words, at $\left.N<N_{\text {cr }}\right)$, where $\left(\mu_{\mathrm{cr}}, N_{\mathrm{cr}}\right)$ are coordinates of the SSB bifurcation point in Fig. 1 . At $\mu<\mu_{\mathrm{cr}}\left(N>N_{\mathrm{cr}}\right)$, the symmetric mode is destabi-
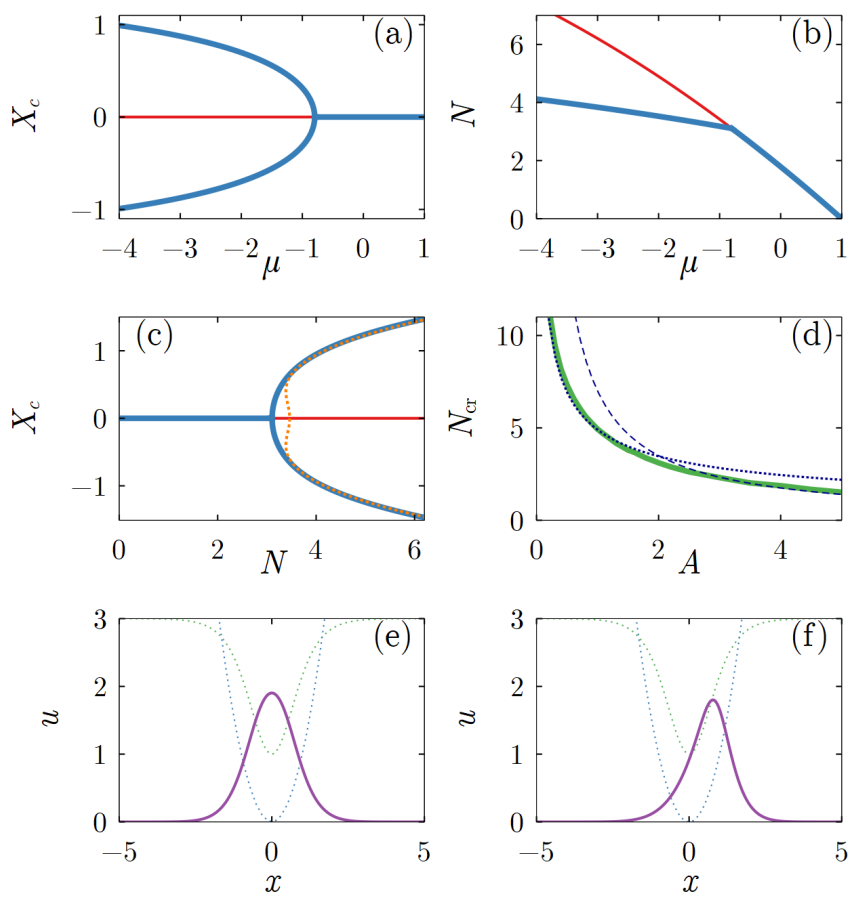

FIG. 1. Panels (a,b,c) show three renditions of the SSB bifurcation for bounded pseudopotential (2) with $A=2$. Stable and unstable solutions correspond to thick blue and thin red segments of the lines, respectively. The dotted line in (c) represents the analytical approximation given by Eq. 13. (d) The critical norm (thick green line), at which the SSB takes place in the presence of bounded pseudopotential (2), vs. depth $A$ of its profile. At $N<N_{\text {cr }}$, no asymmetric modes exist, and symmetric ones are stable. At $N>N_{\text {cr }}$ there exist stable asymmetric modes, while symmetric ones are unstable. The thin dashed and dotted lines depict analytical predictions (6) and (12), which are valid for large $A$ and large $N_{\mathrm{cr}}$, respectively. Indefinite coefficient in Eq. (6) is chosen as const $\approx 7$ to provide for the best fit. (e) and (f) Typical examples of symmetric and asymmetric modes at $A=2$ and $\mu=-2$. The thin dotted lines depict the parabolic potential and pseudopotential $P(x)$. For all panels, the strength of the HO trapping is $\omega=\sqrt{2}$.

lized by a single negative eigenvalue in the spectrum of $\mathcal{L}$, while the emerging asymmetric modes are stable.

Direct simulations of the evolution of symmetric and asymmetric modes in the framework of time-dependent GPE (1) confirm the predictions of the linear-stability analysis. As a representative example, in Fig. 2 we display the behavior of an unstable symmetric mode. The input was chosen in the form of the unstable mode, $u(x)$, with a small initial perturbation added to it [in particular, an appropriate perturbation can be introduced merely by multiplying $u(x)$ by 1.001]. Even this mild (and symmetric) perturbation rapidly triggers strong dynamical instability, which spontaneously breaks the symmetry of the initial mode, shifting sideward (to the left, in Fig. 2), thus creating an asymmetric state with irregular internal oscillations, which do not destroy the emerging 


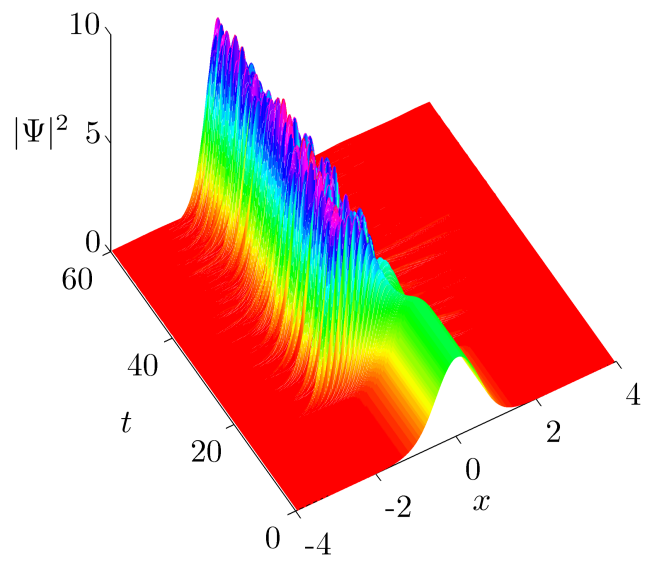

FIG. 2. Evolution $|\Psi(x, t)|^{2}$ of an unstable symmetric mode in bounded pseudopotential (2) with $A=2, \mu=-2$ and $N \approx 4.90$.

asymmetric mode.

Now we aim to demonstrate that the SSB scenario can be essentially altered by taking another pseudopotential, which is also expulsive, but with an unbounded shape, unlike the bounded one in Eq. (2):

$$
P(x)=1+A x^{2} .
$$

Strictly speaking, the indefinite growth of the local nonlinearity strength at $|x| \rightarrow \infty$, which is implied by Eq. (8), is not possible, but in practical terms it may be truncated at values of $|x|$ which are much larger than the size of the trapped mode 42 . In the physical settings, this pseudopotential can be implemented by means of the same approach as the one defined by Eq. (2).

On the contrary to the case of pseudopotential (2), the symmetric state undergoes the inverted pitchfork SSB bifurcation with the increase of $N$. The corresponding SSB diagrams are displayed in Fig. 3(a,b,c), where the panels with different renditions of the SSB bifurcation are organized in the same way as in Fig. 1. In particular, we observe that slope $d N / d \mu$ of the asymmetric branch is positive in this case, hence the VK criterion implies that the branch is unstable. This prediction agrees with the numerical computation of the linear stability eigenvalues, which produces a single negative (unstable) eigenvalue in the spectrum of the linearization operator $\mathcal{L}$ for this branch. As above, the symmetric state is destabilized by the bifurcation (again, with one negative eigenvalue emerging in the spectrum of $\mathcal{L}$ ), when the pair of the unstable asymmetric states merge into the symmetric one, at $N=N_{\text {cr }}$. Furthermore, at $N<N_{\text {cr }}$ the symmetric state is not a ground state, but only a metastable one, which is made clear by both numerical simulations and analytical results presented below. The observed change of the slope of $N(\mu)$ dependence and the destabilization of the symmetric and asymmetric modes is consistent with the rigorous treatment developed in Ref. 17. Spatial shapes of solutions in the unbounded potential are
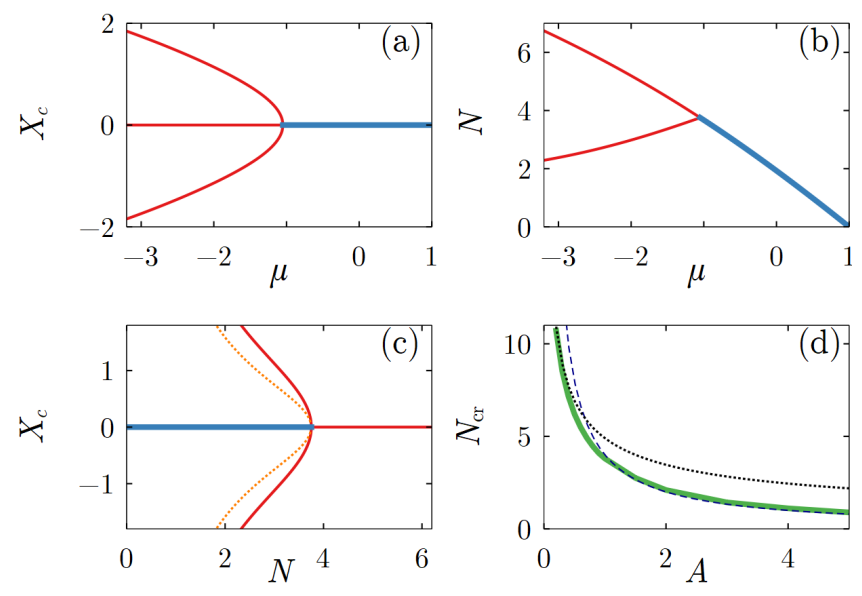

FIG. 3. The SSB bifurcation for the unbounded pseudopotential (8), with $A=1$ and $\omega=\sqrt{2}$. Panels (a-d) are organized as in Fig. 1. Dotted line in (c) is the analytical prediction 14. For the analytical approximation (6), valid for large $A$ the dashed line in $(\mathrm{d})$ ], const $\approx 5$ is chosen to provide for the best fit.

qualitatively similar to those in the bounded one [see Fig. [1(e,f)], therefore they are not shown in Fig. 3.

Dynamics of unstable asymmetric states in the unbounded pseudopotential is sensitive to the choice of initial perturbations. In particular, multiplying an unstable asymmetric mode $u(x)$ with $X_{c}<0$ by $e^{i k x}$, with right-directed kick $0<k \ll 1$, one triggers oscillations of the solution's center between $X_{c}$ and $-X_{c}$, as shown in Fig. 4 (a). On the other hand, the application of small $k<0$ naturally initiates drift of the quasi-soliton further to the left, where the growing attractive nonlinearity makes the solution very narrow, driving the growth of its amplitude, see Fig. 4(b). A similar scenario, i.e., spontaneous sideward drift, is typical for unstable symmetric modes.

\section{THE ANALYTICAL APPROACH}

In addition to the simple analytical result given by Eq. (6), which is relevant for small $N$, an analytical approximation can be developed for large $N$. In this case, strong nonlinearity makes the soliton, with its center located at a point with coordinate $X$, very narrow, hence Eq. (4) gives rise to the approximate solution,

$$
u_{0}(x)=\sqrt{-\frac{2 \mu}{P(X)}} \operatorname{sech}(\sqrt{-\mu}(x-X)), N=\frac{4 \sqrt{-\mu}}{P(X)},
$$

Then, the substitution of this in Eq. (3) yields an effective soliton's potential energy,

$$
U(X)=-\left(N^{3} / 48\right)(P(X))^{2}+\left(\omega^{2} N / 2\right) X^{2},
$$

which predicts equilibria, $X=X_{c}$ [see Eq. (50]), at points with $U^{\prime}\left(X_{c}\right)=0$. 

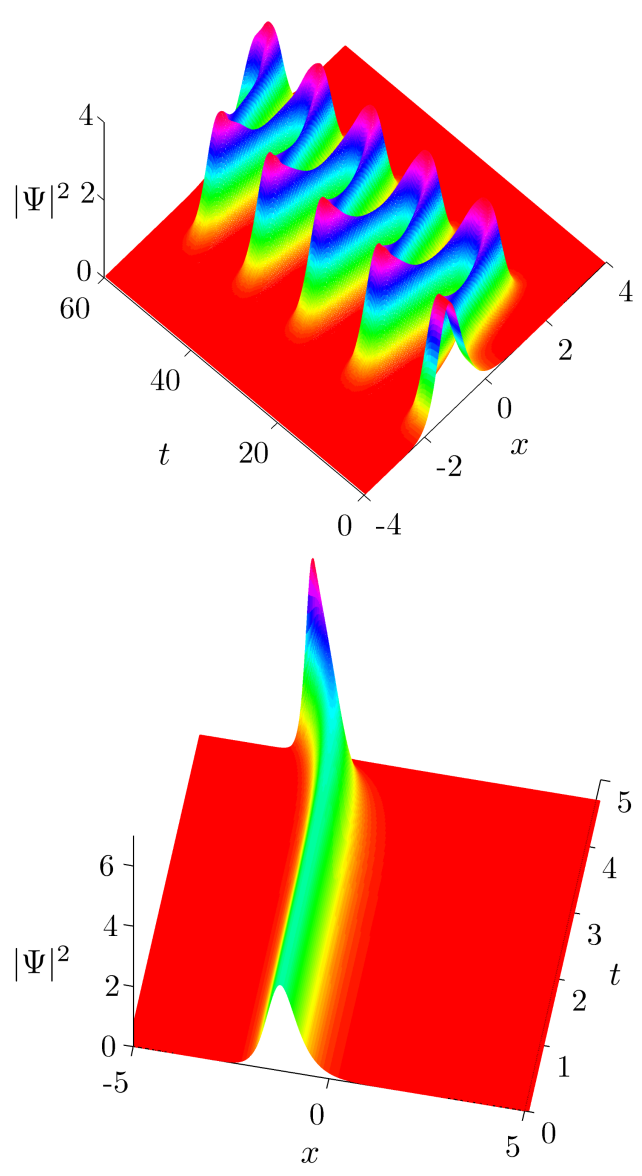

FIG. 4. Different dynamics for the same unstable asymmetric mode, with $\mu=-2$, subjected to different initial perturbations, under the action of unbounded pseudopotential (8) with $A=1$.

First, for vanishingly small $X$, the expansion of potential 10 yields, for both forms of $P(A)$ defined by Eqs. (2) and (8),

$U(X)=-\left(N^{3} / 48\right)+(N / 2)\left[\omega^{2}-\left(A N^{2} / 12\right)\right] X^{2}+\mathcal{O}\left(X^{4}\right)$.

From here it follows that the SSB bifurcations, which implies a transition from the stable to unstable equilibrium at $X=0$, occurs at

$$
N_{\text {cr }}=2 \omega \sqrt{3 / A}
$$

Further, at $N>N_{\text {cr }}$ the substitution of the bounded nonlinearity-modulation profile 2 in equation $d U /\left.d X\right|_{X=X_{c}}=0$, which follows from Eq. (10), yields an equation predicting a mutually symmetric pair of the asymmetric equilibrium points,

$$
\left(1+A \tanh ^{2} X_{c}\right) \frac{\tanh X_{c}}{\cosh ^{2} X_{c}}=\frac{12 \omega^{2}}{A N^{2}} X_{c} .
$$

Then, straightforward analysis demonstrates that the asymmetric equilibria are always local minima of potential 10, with $d^{2} U /\left.d X^{2}\right|_{X=X_{c}}>0$, hence this pair of the equilibrium points are stable, in accordance with the numerical findings presented above.

The validity of approximations 12 and $(13)$ for the bounded pseudopotential is illustrated in Figs. 1 (d) and 1.(c), respectively. For sufficiently large $N$, the analytical predictions (dotted lines) are practically identical to their numerical counterparts.

For the unbounded modulation profile (8), the equilibrium condition predicts the existence of unstable asymmetric equilibria [local potential maxima, with $\left.d^{2} U /\left.d X^{2}\right|_{X=X_{c}}<0\right]$ in the subcritical region, at $N<$ $N_{\text {cr: }}$ :

$$
X_{c}^{2}=A^{-1}\left(N_{\mathrm{cr}}^{2} / N^{2}-1\right),
$$

which explains the numerical findings reported above, see the comparison with numerical results in Fig. 3(c,d). Furthermore, effective potential (10) is always globally expulsive in the present case, with term - $\left(N^{3} / 48\right) A^{2} X^{4}$ dominating at large $|X|$. This fact implies that the model with the unbounded nonlinear potential does not have a ground state, the symmetric one being metastable at $N<N_{\text {cr }}$, as mentioned above.

\section{CONCLUSION}

For the first time, to the best of our knowledge, we have demonstrated that the competition between singlewell linear and nonlinear potentials enables the effect of the SSB (spontaneous symmetry breaking) of nonlinear modes. The reported SSB bifurcation scenario is rather unusual since, contrary to most of the previously reported settings where the symmetry breaking has been encountered, our system does not require any double- or multi-well potential. With the increase of the solution's norm, $N$, the bifurcation occurs at the critical value, $N=N_{\text {cr }}$. There two different bifurcation scenarios, depending on the form of the nonlinear pseudopotential. If it is bounded, the bifurcation is direct, destabilizing the symmetric ground state and producing the pair of asymmetric ones. On the other hand, the bifurcation is inverted, leading to the merger of the pair of unstable asymmetric states into the symmetric one, for unbounded pseudopotentials. Parallel to the systematically collected numerical results, basic results are accurately explained by the simple analytical approximation, which represents the self-trapped modes as narrow solitons. The settings explored in this work may be realized in BEC and nonlinear optics.

As an extension of the present analysis, it may be relevant to consider a setting with the competition between a confining nonlinear potential and expulsive linear one, as a generalization of the antiwaveguiding settings in the self-focusing media; in particular, the analytical approximation developed here may be relevant in that case too. A challenging possibility is to consider a two-dimensional version of the present model, which, as well as the onedimensional system, can be realized in BEC and non- 
linear optics (using bulk waveguides, in the latter case). Finally, while the present work is focused on the symmetry breaking of the nodeless ground states, it is also relevant to extend the analysis for the excited states, in which SSB effects may be expected too.

\section{ACKNOWLEDGMENTS}

Authors are grateful to K. A. Kolesnikova for the help in numerical computations. The research of G.L.A,
M.E.L and D.A.Z. was supported by Russian Science Foundation (Grant No. 17-11-01004). The work of D.A.Z. was also supported by Government of Russian Federation (Grant 08-08). The work of B.A.M. is supported, in part, by Israel Science Foundation (Grant No. $1287 / 17)$.
[1] F. Grossmann, T. Dittrich, P. Jung, and P. Hänggi, Coherent destruction of tunneling, Phys. Rev. Lett. 67, 516519 (1991); F. Cooper, A. Khare, and U. Sukhatme, Supersymmetry and quantum mechanics, Phys. Rep. 251, 268-385 (1995); T. Schumm, S. Hofferberth, L. M. Andersson, S. Wildermuth, S. Groth, I. Bar-Joseph, J. Schmiedmayer, and P. Kruger, Matter-wave interferometry in a double well on an atom chip, Nat. Phys. 1, 57-62 (2005); R. Gati and M. K. Oberthaler, A bosonic Josephson junction, J. Phys. B: At. Mol. Opt. Phys. 40, R61-R89 (2007).

[2] G. J. Milburn, J. Corney, E. M. Wright, and D. F. Walls, Quantum dynamics of an atomic Bose-Einstein condensate in a double-well potential, Phys. Rev. A 55, 43184324 (1997).

[3] A. Smerzi, S. Fantoni, S. Giovanazzi, and S. R. Shenoy, Quantum coherent atomic tunneling between two trapped Bose-Einstein condensates, Phys. Rev. Lett. 79, 4950-4953 (1997).

[4] V. M. Pérez-García, H. Michinel, and H. Herrero, BoseEinstein solitons in highly asymmetric traps, Phys. Rev. A 57, 3837-3842 (1998).

[5] R. D'Agosta and C. Presilla, States without a linear counterpart in Bose-Einstein condensates, Phys. Rev. A 65, 043609 (2002).

[6] R. K. Jackson and M. I. Weinstein, Geometric analysis of bifurcation and symmetry breaking in a Gross-Pitaevskii equation, J. Stat. Phys. 116, 881-905 (2004).

[7] P. G. Kevrekidis, Z. Chen, B.A. Malomed, D. J. Frantzeskakis, and M. I. Weinstein, Spontaneous symmetry breaking in photonic lattices: Theory and experiment, Phys. Lett. A 340, 275-280 (2005).

[8] G. Theocharis, P. G. Kevrekidis, D. J. Frantzeskakis, and P. Schmelcher, Symmetry breaking in symmetric and asymmetric double-well potentials, Phys. Rev. E 74, 056608 (2006).

[9] T. Kapitula, P. G. Kevrekidis, and Z. Chen, Three is a crowd: Solitary waves in photorefractive media with three potential wells, SIAM J. Appl. Dyn. Syst. 5(4), 598-633 (2006).

[10] G. L. Alfimov and D. A. Zezyulin, Nonlinear modes for the Gross-Pitaevskii equation - a demonstrative computation approach, Nonlinearity 20, 2075-2092 (2007).

[11] M. Matuszewski, B.A. Malomed, M. Trippenbach, Spontaneous symmetry breaking of solitons trapped in a double-channel potential, Phys. Rev, A 75, 063621 (2007).
[12] E. W. Kirr, P. G. Kevrekidis, E. Shlizerman, and M. I. Weinstein, Symmetry-breaking bifurcation in nonlinear Schrödinger/Gross-Pitaevskii equations, SIAM J. Math. Anal. 40, 56-604 (2008).

[13] D. A. Zezyulin, G. L. Alfimov, V. V. Konotop, and V. M. Pérez-García, Stability of excited states of a BoseEinstein condensate in an anharmonic trap, Phys. Rev. A 78, 013606 (2008).

[14] A. Sacchetti, Universal Critical Power for Nonlinear Schrödinger Equations with a Symmetric Double Well Potential, Phys. Rev. Lett. 103, 194101 (2009).

[15] H. A. Cruz, V. A. Brazhnyi, V. V. Konotop, and M. Salerno, One-dimensional delocalizing transitions of matter waves in optical lattices, Physica D 238, 1372-1387 (2009).

[16] E. W. Kirr, P. G. Kevrekidis, D. E. Pelinovsky, Symmetry-breaking bifurcation in the nonlinear Schrödinger equation with symmetric potentials, Comm. Math. Phys. 308, 795-844 (2011).

[17] J. Yang, Stability analysis for pitchfork bifurcations of solitary waves in generalized nonlinear Schrödinger equations, Physica D 244, 50-67 (2013).

[18] C. M. de Sterke, I. V. Kabakova, I. Uddin, J. Jeyaratnam, and B. A. Malomed, Spontaneous symmetry breaking in a double-defect nonlinear grating, Phys. Rev. A 88, 033825 (2013).

[19] E Shamriz, N. Dror, and B. A. Malomed, Spontaneous symmetry breaking in a split potential box, Phys. Rev. E 94, 022211 (2016).

[20] K. B. Zegadlo, N. Dror, M. Trippenbach, M. A. Karpierz, and B. A. Malomed, Spontaneous symmetry breaking of self-trapped and leaky modes in quasi-double-well potentials, Phys. Rev. A 93, 023644 (2016).

[21] J. Yang, Nonlinear Waves in Integrable and Nonintegrable Systems (Society for Industrial and Applied Mathematics: Philadelphia; 2010).

[22] D. Landau and E. M. Lifshitz, Quantum Mechanics (Nauka Publishers, Moscow, 1974).

[23] E. B. Davies, Symmetry breaking in a non-linear Schrödinger equation, Commun. Math. Phys. 64, 191210 (1979).

[24] J. C. Eilbeck, P. S. Lomdahl, and A. C. Scott, The discrete self-trapping equation, Physica D 16, 318-338 (1985).

[25] W. A. Harrison, Pseudopotentials in the Theory of Metals (Benjamin, New York, 1966).

[26] J. Hukriede, D. Runde, and D. Kip, Fabrication and ap- 
plication of holographic Bragg gratings in lithium niobate channel waveguides, J. Phys. D 36, R1-R16 (2003).

[27] D. M. Bauer, M. Lettner, C. Vo, G. Rempe and S. Dürr, Control of a magnetic Feshbach resonance with laser light, Nature Phys. 5, 339-342 (2009).

[28] R. Yamazaki, S. Taie, S. Sugawa, and Y. Takahashi, Submicron spatial modulation of an interatomic interaction in a Bose-Einstein condensate, Phys. Rev. Lett. 105, 050405 (2010).

[29] L. W. Clark, L.-C. Ha, C.-Y. Xu, and C. Chin, Quantum dynamics with spatiotemporal control of interactions in a stable Bose-Einstein condensate, Phys. Rev. Lett. 115, 153301 (2015).

[30] K. Henderson, C. Ryu, C. MacCormick,and M. G. Boshier, Experimental demonstration of painting arbitrary and dynamic potentials for Bose-Einstein condensates, New J. Phys. 11, 043030 (2009).

[31] Y. V. Kartashov, B. A. Malomed, and L. Torner, Solitons in nonlinear lattices, Rev. Mod. Phys. 83, 247-306 (2011).

[32] T. Mayteevarunyoo, B. A. Malomed, and G. Dong, Spontaneous symmetry breaking in a nonlinear double-well structure, Phys. Rev. A 78, 053601 (2008).

[33] A. Acus, B. A. Malomed, and Y. Shnir, Spontaneous symmetry breaking of binary fields in a nonlinear doublewell structure, Physica D 241, 987-1002 (2012).

[34] N. Dror and B. A. Malomed, Solitons and vortices in nonlinear potential wells, J. Optics 16, 014003 (2016).

[35] D. Bortman-Arbiv, A. D. Wilson-Gordon, and H. Friedmann, Strong parametric amplification by spatial solitoninduced cloning of transverse beam profiles in an alloptical antiwaveguide, Phys. Rev. A 63, 031801 (2001); A. Kaplan, B. V. Gizsin, and B. A. Malomed, Stable propagation and all-optical switching in planar waveguide-antiwaveguide periodic structures, J. Opt. Soc. Am. B 19, 522-528 (2002).

[36] G. Iooss and D. D. Joseph, Elementary Stability Bifurcation Theory (Springer, New York, 1980).

[37] L. P. Pitaevskii and A. Stringari, Bose-Einstein Condensation (Clarendon Press, Oxford, 2003).

[38] M. Vakhitov and A. Kolokolov, Stationary solutions of the wave equation in a medium with nonlinearity saturation, Radiophys. Quant. Electron. 16, 783-789 (1973).

[39] L. Bergé, Wave collapse in physics: principles and ap- plications to light and plasma waves, Phys. Rep. 303 , 259-370 (1998).

[40] G. Fibich, The Nonlinear Schrödinger Equation: Singular Solutions and Optical Collapse (Springer: Heidelberg, 2015).

[41] M. Edwards and K. Burnett, Numerical solution of the nonlinear Schrödinger equation for small samples of trapped neutral atoms, Phys. Rev. A 51, 1382-138 (1995); P. A. Ruprecht, M. J. Holland, K. Burnett, and M. Edwards, Time-dependent solution of the nonlinear Schrödinger equation for Bose-condensed trapped neutral atoms, Phys. Rev. A 51, 4704-4711 (1995); F. Dalfovo and S. Stringari, Bosons in anisotropic traps: Ground state and vortices, Phys. Rev. A 53, 2477-2485 (1996); V. I. Yukalov, E. P. Yukalova, and V. S. Bagnato, Non-ground-state Bose-Einstein condensates of trapped atoms, Phys. Rev. A 56, 4845 (1997); M. Kunze, T. Küpper, V. K. Mezentsev, E. G. Shapiro, and S. Turitsyn,Nonlinear solitary waves with Gaussian tails, Physica D 128, 273-295 (1999); Yu. S. Kivshar, T. J. Alexander, and S. K. Turitsyn, Nonlinear modes of a macroscopic quantum oscillator, Phys. Lett. A 278, 225-230 (2001); L. D. Carr, J. N. Kutz, and W. P. Reinhardt, Stability of stationary states in the cubic nonlinear Schrödinger equation: Applications to the Bose-Einstein condensate, Phys. Rev. E 63, 066604 (2001); V. I. Yukalov, E. P. Yukalova, and V. S. Bagnato, Nonlinear coherent modes of trapped Bose-Einstein condensates, Phys. Rev. A 66 , 043602 (2002); R. D'Agosta, B. A. Malomed, and C. Presilla, Stationary states of Bose-Einstein condensates in single-and multi-well trapping potentials, Laser Phys. 12, 37-42 (2002); V. V. Konotop and P. G. Kevrekidis, Bohr-Sommerfeld Quantization Condition for the GrossPitaevskii Equation, Phys. Rev. Lett. 91, 230402 (2003); P. G. Kevrekidis, V. V. Konotop, A. Rodrigues, and D. J. Frantzeskakis, Dynamic generation of matter solitons from linear states via time-dependent scattering lengths, J. Phys. B: At. Mol. Opt. Phys 38, 1173-1188 (2005).

[42] O. V. Borovkova, Y. V. Kartashov, B. A. Malomed, and L. Torner, Algebraic bright and vortex solitons in defocusing media, Opt. Lett. 36, 3088-3090 (2011). 